\title{
ST-Segment Elevation Associated with Mobitz II Atrioventricular Block During Transseptal Puncture for Atrial Fibrillation Ablation
}

Paula Damasco do Vale 1,*, Lívia Teixeira Martins e Silva², Jairo Macedo da Rocha ${ }^{1,2}$ Carla Septimio Margalho ${ }^{1,2}$, Henrique César de Almeida Maia ${ }^{1,2}$

\section{ORCID IDs}

Vale PD (1) https://orcid.org/0000-0003-0244-5524

Silva LTMS (10) https://orcid.org/0000-0002-8282-442X
Rocha JM (1) https://orcid.org/0000-0002-8308-4398

Margalho CS (1) https://orcid.org/0000-0003-4844-7752

Maia HCA (D) https://orcid.org/0000-0001-9215-9585

\begin{abstract}
Pulmonary veins electrical isolation as an invasive treatment of atrial fibrillation has been widely used in electrophysiology laboratories. This case report presents a rare and transient complication, during transseptal puncture for atrial fibrillation ablation. ST-segment elevation, hypotension and bradyarrhythmia related to catheterization were observed despite cineangiocoronariography without obstructive lesions. Clinical stability was achieved after administration of intravenous atropine and saline solution. It is speculated that the phenomenon is attributed to an increased vagal tone after the mechanical effect of transseptal puncture in the interatrial vagal network. The procedure was completed despite the phenomenon.
\end{abstract}

KEYWORDS: Atrial fibrillation; Punctures; Electrocardiography; Catheter ablation.

1. Instituto Hospital de Base do Distrito Federal - Brasília/DF - Brazil.

2. Ritmocardio Serviço de Arritmia e Eletrofisiologia de Brasília - Brasília/DF - Brazil.

*Corresponding author: pauladamasco@hotmail.com

Received: Oct 07, 2020 | Accepted: Nov 20, 2020 


\section{INTRODUCTION}

Access to the left atrium by transseptal puncture is a common practice in electrophysiology laboratories in the treatment by radiofrequency of cardiac arrhythmias. The ST segment elevation during transseptal puncture is mostly described as a sudden and transient alteration on the electrocardiogram, accompanied or not by symptoms with vagal action characteristics. The main pathophysiological mechanisms are vasospasm, due to the imbalance in autonomic innervation; coronary hypoperfusion secondary to reflex action; or air embolism. The incidence of this phenomenon is not well defined, and its description comes from case reports or retrospective records. Despite the diversity of clinical findings in the literature, there are two characteristics in common in the published series: the transience of the phenomenon and the normal angiographic findings.

\section{CASE REPORT}

A 67-year-old man with paroxysmal atrial fibrillation refractory to the use of antiarrhythmics, a candidate for radiofrequency ablation, received oral anticoagulation for three weeks before the procedure, maintaining the international normalized ratio (INR) between 2 and 3, and underwent transesophageal echocardiography, who demonstrated a left atrium with an indexed volume of $39 \mathrm{~mL} / \mathrm{m}^{2}$, a left ventricular ejection fraction of $62 \%$, without an intracavitary thrombus. He underwent sinus rhythm catheter ablation.

After femoral venous access, the Mullins sheath was positioned in the superior vena cava preceded by a Terumo 0.032 "guidewire". The Brockenbrough needle was inserted into the Mullins sheath and positioned approximately $2-3 \mathrm{~cm}$ before the tip of the sheath. With the needle, it was gradually removed from the superior vena cava to the right atrium under fluoroscopy in anteroposterior (AP) projection, taking care that the Brockenbrough needle pointed to the 3 o'clock position. The set descended from the superior vena cava to the right atrium and then along the thick part of the interatrial septum. There was a fall movement during the exit of the superior vena cava and the interatrial septum, and then this same set was gradually removed until a second fall was observed, when the needle was positioned in the oval fossa. The needle was rotated clockwise to the 4-5 o'clock position and its location confirmed by rotating the scopy to the right anterior oblique (RAO) and left anterior oblique (LAO) positions.

The confirmation of the proper position was defined by the operator's opposition and by not pointing to the left (posterior) or to the right (anterior). In addition, the needle was positioned posteriorly and inferiorly to the plane of the aorta. In the right side view, it was turned posteriorly between the spine and the catheter positioned in the coronary sinus (Fig. 1). In LAO, it was between the final two thirds between the curvature of the right atrium and the coronary sinus catheter (Fig. 2).

In this position, the septum was punctured, and, right after the transseptal puncture with a drop-off signal observed in fluoroscopy, it was recorded on the electrophysiology polygraph (EP-TRACER V 1.0 ${ }^{\circledR}$ ) elevation of the ST segment of $4 \mathrm{~mm}$ in DIII, in addition to junctional bradycardia with a heart rate of $35 \mathrm{bpm}$, blood pressure $93 \times 37 \mathrm{mmHg}$ and sweating. The junctional rhythm evolved to 2:1 conduction, followed by second-degree atrioventricular block (AVB) type Mobitz II (Fig. 3), with an infusion of atropine to reverse the sinus rhythm (Fig. 4) and saline to maintain blood pressure levels.

The disappearance of ST elevation occurred after resolution of the clinical picture. The cinecoronariography performed immediately after the onset of the phenomenon proved to be normal. After the normalization of the ST segment with hemodynamic stability and the absence of coronary changes, the procedure was successfully concluded. 


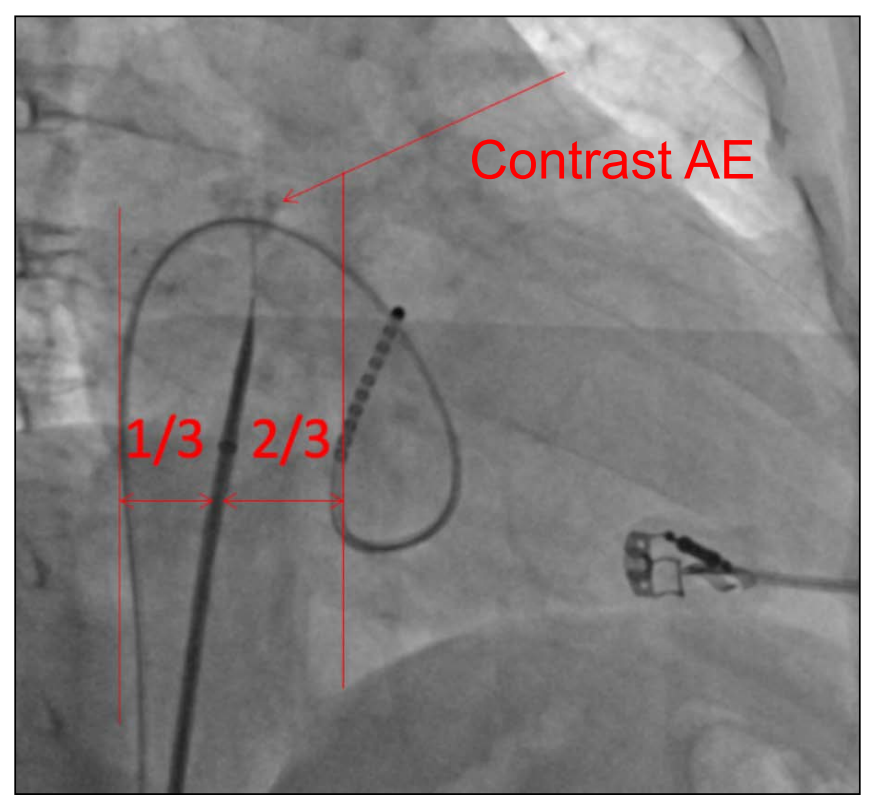

Figure 1. Right anterior oblique position. Contrast in the LA (AE: Left Atrium).

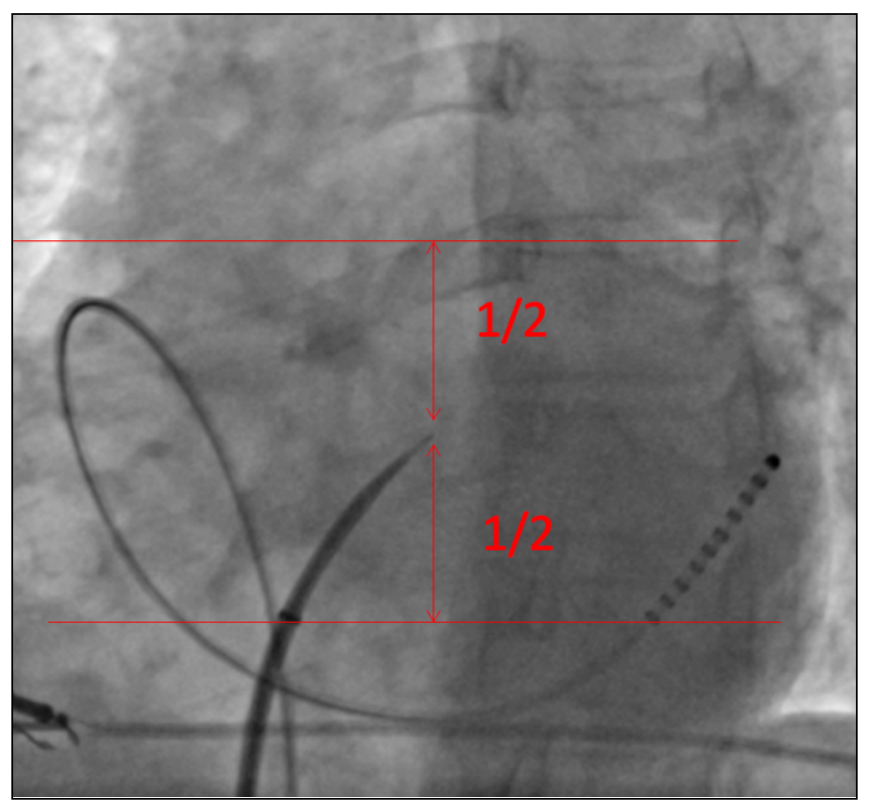

Figure 2. Left anterior oblique position.

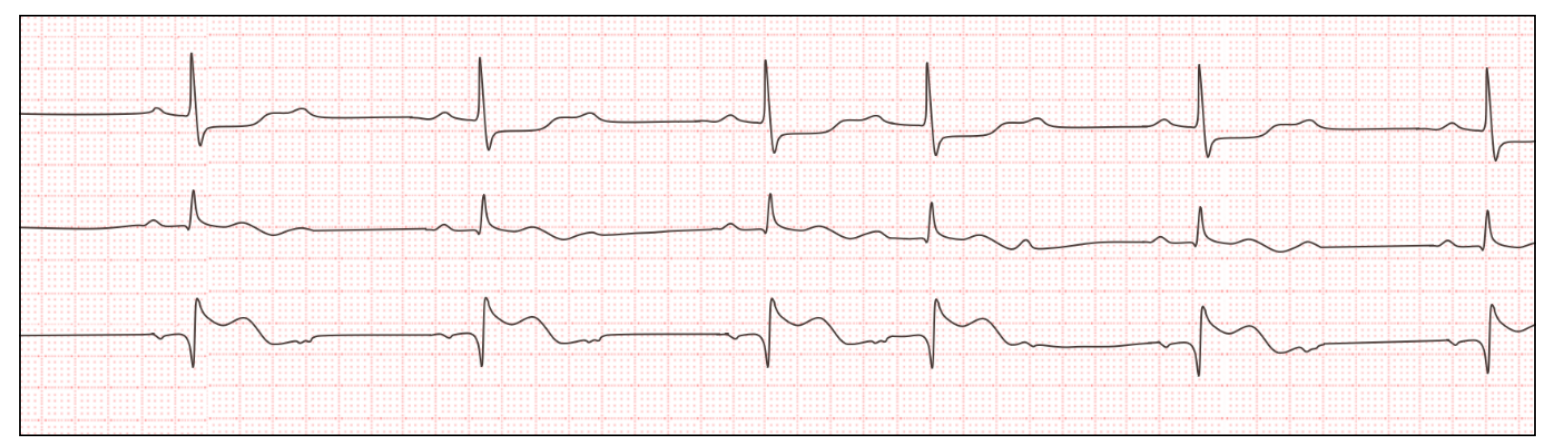

Figure 3. Electrocardiogram showing 2: 1 conduction, followed by Mobitz II atrioventricular block after transseptal puncture. 


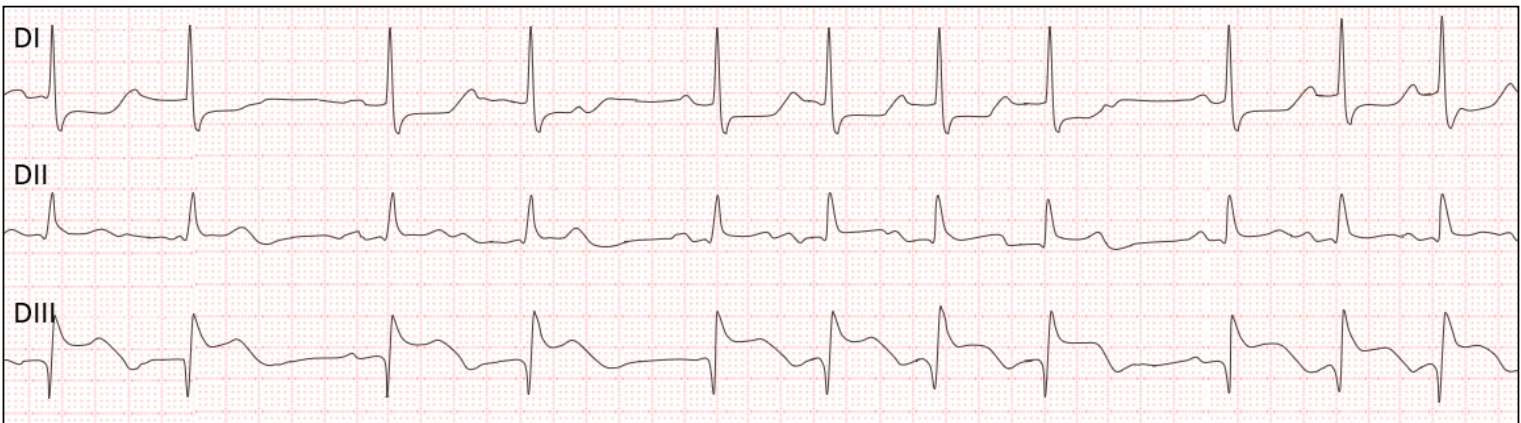

Figure 4. Reversal of Mobitz II atrioventricular block after atropine infusion.

\section{DISCUSSION}

For the treatment of arrhythmias related to the left atrium, access to the left chambers is necessary, with the transseptal puncture being the conventional method. This procedure is not without risks and may occur complications such as thromboembolism, gas embolism and coronary artery spasm, may occur, in addition to hemopericardium, cardiac tamponade and bleeding from the puncture of adjacent structures ${ }^{1-3}$.

There are descriptions of sudden and transient ST-segment elevation in the lower leads, associated or not with bradycardia and arterial hypotension, related to transseptal puncture ${ }^{2,4-7}$. Most of the marked changes on the surface electrocardiogram are accompanied by symptoms with vagal action characteristics. The analysis of the cases described in the literature shows that, in almost its entirety, changes in the ST segment occur during transseptal puncture, however there are some records of the phenomenon before it and during the manipulation of the pulmonary veins.

The change in the classic ST segment is constantly linked to electrocardiographic leads, with a pattern suggestive of ischemia of the lower wall. The mechanism and the underlying pathophysiology of ST segment changes are not well established. At least three possible mechanisms for this phenomenon are described: the reflex action similar to the BezoldJarisch reflex, coronary vasospasm and the occurrence of coronary microembolism.

The Bezold-Jarisch reflex is an inhibitory reflex originating from sensory cardiac receptors with vagal afferent fibers that are influenced by chemical or mechanical stimuli. This stimulation increases parasympathetic activity and inhibits sympathetic activity, producing bradycardia, vasodilation and hypotension ${ }^{5,8,9}$. Right coronary dilation, hypotension and bradycardia would result in coronary hypoperfusion and regional and transient myocardial ischemia, which can generate electrocardiographic changes in the ST segment during transseptal puncture ${ }^{2,5}$, however, the hypothesis of the reflex similar to that of Bezold-Jarisch leading to ST change does not explain the fact that, after a few minutes of septal puncture, the clinical and electrocardiographic changes disappear, despite the continuous stimulation of the septum by the presence of the sheath used during the puncture.

The hypothesis that the electrocardiographic and clinical picture observed during the puncture is secondary to vasospasm of the coronary artery is based on the presumption that manipulation of the intrasseptal plexuses and left atrial ganglia by the needle and transeptal sheath may cause imbalance in the autonomic innervation and lead to the hypervagotonic state $^{3,7,10}$. The induced hypervagotonia would result in the release of acetylcholine, which would cause spasm in the coronary circulation ${ }^{6}$. The right coronary artery is more susceptible to cholinergic action and vasospasm ${ }^{3}$.

Another possible mechanism is the introduction of air emboli through the transeptal sheath, which would migrate to the coronary artery, resulting in acute gas embolism ${ }^{3,6,11}$, however, there are several explanations that make the procedure unlikely:

- The coronary arteries have a predominant diastolic perfusion and origin perpendicular to the laminar flow at the root of the aorta;

- Thrombi of the left atrium usually go to the cerebral circulation and less commonly to the coronary arteries;

- Changes in the ST segment have a predominant location in the lower wall, but air embolism should occur with an identical predominance between the right and left coronary arteries; 
- Embolism also does not explain the occurrence of changes in patients who have an elevation of the ST segment before transseptal puncture;

- Most coronary angiographies performed immediately after the ST segment changes were found to be normal ${ }^{2,3,7,8,11}$.

None of these mechanisms explains the changes found during catheterization per se. Regardless of the presence or absence of clinical symptoms, it is a self-limited and short-lived phenomenon, in which catheter ablation can be safely concluded and without other complications. Therefore, the occurrence of this phenomenon should not result in the interruption of the ablation procedure.

\section{CONCLUSION}

ST-segment elevation is a phenomenon that affects mainly the lower wall during transeptal catheterization. Only shortterm electrocardiographic changes can occur without clinically evident symptoms. Even when associated with hypotension and bradycardia, spontaneous recovery occurs after a few minutes and requires little therapeutic intervention. It is a selflimited event, and the catheter ablation procedure can be safely concluded, despite the occurrence of the phenomenon.

\section{REFERENCES}

1. Haegeli LM, Wolber T, Ercin E, Altwegg L, Krasniqi N, Novak PG, et al. Double transseptal puncture for catheter ablation of atrial fibrillation: Safety of the technique and its use in the outpatient setting. Cardiol Res Pract. 2010;2010:295297. https://doi. org/10.4061/2010/295297

2. Glowniak A, Tarkowski A, Jaroszyńska A, Jankiewicz M, Wysokiński A. Transient symptomatic ST-segment elevation following transseptal puncture for pulmonary vein isolation. Prog Med. 2015;28(8):567-9.

3. Arita T, Kubota S, Okamoto K, Kuma F, Nakasuga K, Koga H, et al. Bezold-Jarisch-like reflex during Brockenbrough's procedure for radiofrequency catheter ablation of focal left atrial fibrillation: Report of two cases. J Interv Card Electrophysiol. 2003;8:195-202. https://doi.org/10.1023/A:1023917221763

4. Cheng YL, Dong JZ, Liu XP, Long DY, Fang DP, Yu RH, et al. Transient ST-segment elevation after transseptal puncture for atrial fibrillation ablation in two cases. Chin Med J (Engl). 2012;125(5):941-4. https://doi.org/10.3760/cma.j.issn.0366-6999.2012.05.037

5. Simon RDB, Gill JS. Coronary ischemia induced by radiofrequency ablation in the left atrium. J Cardiovasc Electrophysiol. 2003;14(2):186-90. https://doi.org/10.1046/j.1540-8167.2003.02474.x

6. Tang RB, Dong JZ, Long DY, Yu RH, Liu XP, Cheng YL, et al. Incidence and clinical characteristics of transient ST-T elevation during transseptal catheterization for atrial fibrillation ablation. Europace. 2015;17(4):579-83. https://doi.org/10.1093/europace/euu278

7. Le BH, Black JN, Huang SKS. Transient ST-segment elevation during transseptal catheterization for atrial fibrillation ablation. Texas Hear Inst J. 2010;37(6):717-21.

8. Warltier DC, Campagna JA, Carter C. Clinical relevance of the Bezold-Jarisch reflex. Anesthesiology. 2003;98:1250-60. https://doi. org/10.1097/00000542-200305000-00030

9. Ishigaki D, Arimoto T, Iwayama T, Daisuke K, Yoshinori Y, Jobi N, et al. ST-segment elevation and ventricular fibrillation shortly after transseptal puncture for left atrial catheter ablation. J Arrhythmia. 2013;29(5):296-9. https://doi.org/10.1016/j.joa.2013.03.004

10. Efremidis M, Letsas KP, Xydonas S, Koutras K, Sideris A, Kardaras F. ECG findings of acute myocardial infarction and atrioventricular block during a transseptal procedure for left atrial ablation. Hell J Cardiol. 2008;49:284-7.

11. Risius T, Lewalter T, Lüderitz B, Schwab JO, Spitzer S, Schmitt C, et al. Transient ST-segment elevation during pulmonary vein ablation using circumferential coiled microelectrodes in a prospective multi-centre study. Europace. 2006;8(3):178-81. https://doi. org/10.1093/europace/euj013 